\title{
Flexible PDMS microparticles to mimic RBCs in blood particulate analogue fluids
}

\author{
D. Pinho ${ }^{\mathrm{a}, \mathrm{b}, \mathrm{e}, \mathrm{f}, 1}$, B.N. Muñoz-Sánchez ${ }^{\mathrm{c}, 1}$, C.F. Anes ${ }^{\mathrm{a}, 1}$, E.J. Vega ${ }^{\mathrm{d}}$, R. Lima ${ }^{\mathrm{e}, \mathrm{f}, *}$ \\ ${ }^{a}$ Research Centre in Digitalization and Intelligent Robotics (CeDRI), Instituto Politécnico de Bragança, Campus de Santa Apolónia, 5300-253 Bragança, \\ Portugal \\ ${ }^{\mathrm{b}}$ INL-International Iberian Nanotechnology Laboratory, Av. Mestre Jose’ Veiga, 4715-330 Braga, Portugal \\ c Departamento de Ingeniería Aeroespacial y Mecánica de Fluidos, Universidad de Sevilla, E-41092 Sevilla, Spain \\ d Departamento de Ingeniería Mecánica, Energética y de los Materiales and Instituto de Computación Científica Avanzada (ICCAEx), Universidad de \\ Extremadura, E-06006 Badajoz, Spain \\ e MEtRICs, Mechanical Engineering Department, Campus de Azurém, University of Minho, 4800-058 Guimarães, Portugal \\ ${ }^{f}$ Transport Phenomena Research Center, Dep. of Chemical Eng., Eng. Faculty, R. Dr. Roberto Frias, Porto, 4200-465, Portugal
}

\section{A R T I C L E I N F O}

\section{Article history:}

Received 23 June 2019

Revised 13 August 2019

Accepted 14 August 2019

Available online 15 August 2019

\section{Keywords:}

PDMS microparticles

Red blood cells

Deformability and rheology

Cell-free layer

Particulate blood analogue

\begin{abstract}
A B S T R A C T
Polydimethylsiloxane (PDMS) has a wide variety of commercial and industrial applications due to its mechanical and rheological properties in a range similar to the living tissues. In this study, we demonstrate that PDMS can be used to produce deformable microparticles to be integrated in the development of particulate blood analogue fluids. The difficulties associated with the use of in vitro blood make it necessary to perform in vitro experiments of blood flow with blood analogue fluids. However, an ideal analogue must match the rheology of blood at several points, and for that, blood analogue fluids should be a suspension of microparticles with similar properties (size, shape and flexibility) to blood cells, in particular to the red blood cells (RBCs). The microparticles used in this study were produced from a transparent PDMS with crosslinking ratios of 10:1, 8:2 and 6:4; from a black PDMS with a ratio of 1:1 and from a red-pigmented PDMS. Each PDMS microparticles sample was suspended in Dextran 40 to perform deformability assays and cell-free layer analysis in a hyperbolic-shaped microchannel and steady shear viscosity measurements in a rheometer. The proposed microparticles suspensions show a great potential to mimic the structural and rheological properties of RBC suspensions and consequently to develop blood analogue fluids with rheological properties similar to real blood.
\end{abstract}

(c) 2019 Elsevier Ltd. All rights reserved.

\section{Introduction}

The increased development and progress in the microfluidics technologies over the last several years is a result of combined efforts of researchers with expertise in diverse fields such as fluid mechanics, thermodynamics, applied physics, chemistry, materials science, manufacturing, biochemistry, biotechnology and medicine [1-3]. Recent microfluidic and micro-electro-mechanical systems (MEMS) have demonstrated that the high elasticity (flexibility) of polydimethylsiloxane (PDMS) offers unique advantages over more traditional rigid substrate materials such as glass and harder polymers $[1,4]$. Thus, PDMS has become the most widely used siliconbased organic polymer due to its unique mechanical and optical properties, and is generally considered inert, non-toxic and non-

\footnotetext{
* Corresponding author.

E-mail address: rl@dem.uminho.pt (R. Lima).

1 These authors contributed equally to the manuscript.
}

flammable, among other characteristics [3,5-7]. Its applications range from contact lenses and medical devices to shampoos, caulking, lubricating oils and heat-resistant tiles [3,6]. An increasing number of devices are being reported to employ the relative deformability of the low-hardness PDMS in order to facilitate flexible microstructures within composite structures [1], for example: micropumps, PDMS microvalves and flexible micropillar arrays for biological force measurements $[8,9]$, passive microfluidic techniques for plasma separation, cell trapping and cell measurements [1014]; and flexible adaptable fluid lenses [1]. As a subset of microfluidic devices, droplet microfluidics devices are required to produce microscale-diameter droplets of one fluid within a second immiscible carrier fluid.

Droplet generation may be driven using active methods such as piezoelectric actuators and electric fields [15-17], but monodisperse droplets are most commonly formed in continuous pressuredriven nozzles with flow-focusing or co-flowing method [7,16,1820], or T-junction techniques [21-23]. Jian et al. [24] and 
Muñoz-Sánchez et al. [7] proposed a flow-focusing technique where a PDMS precursor was dispersed into microdroplets within a continuous phase.

Stiffness and deformability are particle characteristics that have gained increasing interest, for example, the reversible deformability found in vivo for the red blood cells (RBCs) plays an important role in blood rheology for vessels with diameters smaller than $300 \mu \mathrm{m}$, where the effective viscosity gets reduced due to RBCs migration to the vessel centre, resulting in the formation of a cell-free layer close to the vessels or microdevices walls [2529]. These blood phenomena drive the development of particulate blood analogue fluids with similar properties and flow behaviour $[19,30,31]$. One of the main challenges in the development of these particulate fluids is the production of particles with the required mechanical properties. Several research works [32-34] have proposed synthetic RBC-like particles that mimic the key structural attributes of RBCs as size and shape, but mechanical properties were still far away from that of the real RBCs. Besides, the preparation of the proposed $\mathrm{RBC}$-mimicking particles was expensive and time-consuming, being also the final concentration extremely small.

Thus, the production of monodisperse particles with such an inert elastomer as the PDMS has stimulated great interest of researchers because of their potential applications in biomedicine. Several types of particles with varying stiffness and shapes have been engineered and subjected to narrow channels to investigate their deformability $[26,32,35,36]$, demonstrating high deformability when passing through constricted environments [26,37,38]. Merkel et al. [39] have synthesised hydrogel microparticles with tunable elasticity, which resemble RBCs in size and shape, and by using microfluidic models of vascular constriction, evaluated the behaviour of healthy and diseased RBCs and synthetic mimics of RBCs. Vilanova et al. [40] successfully produced monodisperse silicon droplets to be used as templates for the formation of capsules with potential molecular delivery with controlled release, demonstrating that the mechanical behaviour of the generated capsules could be finely tuned by varying the geometrical parameters. Cui et al. [36] engineered PEG hydrogel particles with adjustable elasticity and with a size similar to human RBCs, and by using atomic force microscopy and a microfluidic blood capillary model that mimics dimensions and pressure differentials of the in vivo environment, they investigated the deformability behaviour of these particles and demonstrated that this behaviour could be tuned to be similar to that of RBCs. Choi et al. [6] by mixing uncured PDMS with distilled water, developed a simple and fast method to produce micro-sized spherical PDMS particles.

All these studies have provided important insights into how particles of various materials can be engineered to have different mechanical properties and biological behaviours, and they reveal the effect of the internal structure of particles on deformability. Nevertheless, the mechanical properties of these microparticles are still far away from those of RBCs, and none of them has shown evidence on how closely they mimic other microscale blood-flow phenomena that happen at both in vivo and in vitro environments.

In this way Pinho et al. [31] and Calejo et al. [30] have developed particulate blood analogues with rigid particles able to show the cell-free layer (CFL) formation downstream of a microchannel contraction and the reproduction of the viscosity curve of rigid RBCs suspensions. However, both studies have suggested that the only way to closely mimic some microscale blood-flow phenomena is by using deformable microparticles. Muñoz-Sánchez et al. [7] proposed a flow-focusing technique to produce micro-sized PDMS flexible spherical particles for biomicrofluidic applications, demonstrating also the ability of the proposed particulate fluid to reproduce the steady shear viscosity curve of ovine RBCs suspended in Dextran 40.
In this work we demonstrate that PDMS is a good option to produce microparticles with close dimensions to human RBCs $(\sim 8 \mu \mathrm{m})$ that deform reversibly, and also that a suspension created with these microparticles presents a steady shear viscosity similar to a suspension of human RBCs. Moreover, the well-known microfluidic phenomena of the CFL is also verified for the developed microparticles. To achieve these main objectives, several samples of PDMS microparticles at different mixture ratios of base/cure agent and pigmentation were obtained by the technique proposed by Muñoz-Sánchez et al. [7]. In order to identify the PDMS mixture with the highest deformability, some assays were performed for the produced microparticles and the results compared with the deformability of human RBCs at similar in vitro conditions. Additionally, by using a rheometer, the steady shear viscosities for the PDMS and RBCs suspensions were obtained and compared. Lastly, by using the same setup from the deformability assays, we checked the cell-free layer (CFL) effect for a PDMS suspension and compared it against RBCs suspensions.

\section{Materials and methods}

\subsection{Flow in microfluidic devices}

As the main focus of this work is about the flow of fluids with suspensions of microparticles, this section shows a brief overview of several important physical phenomena of such fluids flowing through microfluidic devices. The fluid flow behaviour in both macro and microdevices can be characterized by comparing the relation between the values of the inertial and viscous forces. This ratio is known as Reynolds number $\left(R_{e}\right)$. As most of the microfluidic devices have rectangular microchannels the hydraulic diameter $\left(D_{h}\right)$ should be used to determine the dimensionless $R_{e}$ :

$R_{e}=\frac{(\text { inertial force })}{(\text { viscous force })}=\frac{\rho U D_{h}}{\mu}$

where $\rho$ is absolute density of the fluid, $U$ is the average of the fluid, $\mu$ is the viscosity and $D_{h}$ is the hydraulic diameter that can be defined as:

$D_{h}=\frac{4 A}{P}$

where $A$ is the cross-sectional area of the microchannel and $P$ is the wetted perimeter.

In most of microfluidic applications the $R_{e}$ is less than 1 and as a result the viscous effects are the ones that most influence the physical flow phenomena that happens in microfluidic devices. Under this regime, viscous forces are dominant and is known as laminar flow.

There are some simple microchannel geometries where the Navier-Stokes equations can be used to obtain several physical parameters such as: flow velocities, flow rates, shear rates and strain rates [41]. By considering a steady pressure-driven flow in a rectangular microchannel (width, $w$, much bigger than the height, $h$ ) where a pressure drop $\Delta P$ acts over a certain length $(\mathrm{L})$, it is possible to obtain the velocity across the channel by the following equation:

$u(y)=\frac{\Delta P}{2 \mu \mathrm{L}}\left[\left(\frac{h}{2}\right)^{2}-y^{2}\right]$.

In the same way, the flow rate $(\mathrm{Q})$ through a rectangular microchannel of width $w$, can be calculated by the following equation:

$Q=w \int_{0}^{h} u(y) d y=\frac{w h^{3} \Delta P}{12 \mu \mathrm{L}}$.

In microcirculation most often the workings fluids flow through a microchannel under a laminar regime and the pressure, in a 
Table 1

Summary of all working fluids prepared by suspending either PDMS microparticles or human RBCs. Hematocrit (Hct) corresponds to the concentration $(\mathrm{v} / \mathrm{v} \%)$ of RBCs in the sample.

\begin{tabular}{lll}
\hline & Composition of the fluid & Acronym \\
\hline $\begin{array}{l}\text { Suspending medium } \\
\text { Suspensions }\end{array}$ & Dextran 40 (solution in water at 10 wt\%) & Dx 40 \\
of & Dx 40+ PDMS microparticles at 10:1 & $10: 1$ \\
PDMS & Dx 40+PDMS microparticles at 8:2 & $8: 2$ \\
microparticles & Dx 40+PDMS microparticles at 6:4 & $6: 4$ \\
& Dx 40+ Black PDMS microparticles at 1:1 & Black PDMS \\
Blood & Dx 40+ Red-dyed PDMS microparticles at 10:1 PDS \\
fluids & Dx 40+PDMS microparticles at 10:1, 8:2, 6:4 (concentration of 8\%vol) & Final working fluid \\
\hline
\end{tabular}

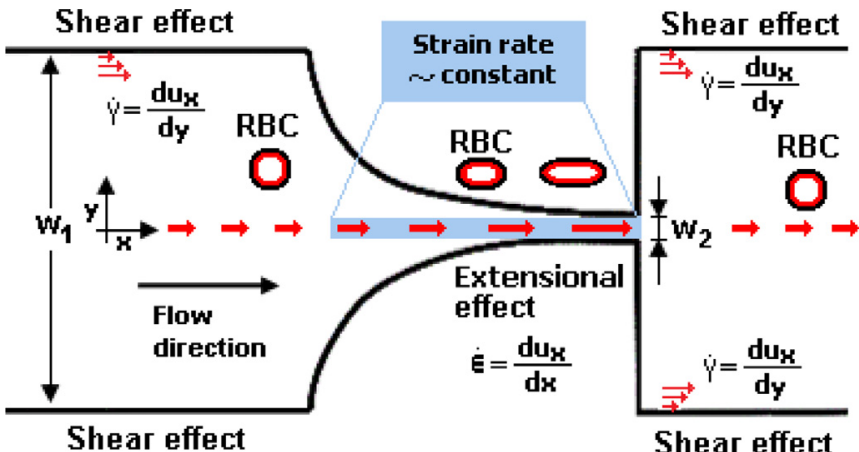

Fig. 1. Schematic of the fluid flow behaviour in a microfluidic hyperbolic-shaped contraction through which the cells and particles flows. This geometry has the ability to generate extensional flows with a homogeneous strain-rate along the centreline of the contraction.

steady flow condition, decreases linearly with the length (L) of channel. Under these conditions the pressure drop is $\triangle P=R Q$, where $Q$ is the flow rate and $R$ is the hydrodynamic resistance of a microvessel [42]. For microfluidic devices with rectangular microchannels the hydrodynamic resistance is given approximately by

$R=\frac{12 \mu \mathrm{L}}{w h^{3}}($ for $h \ll L)$

where $L$ is the length of the channel, $w$ is the width and $h$ is the height. More detailed information about pressure-driven flows in a rectangular microhannels can be found elsewhere [41,43].

In the present study, we have used hyperbolic converging microchannels in order to investigate both microparticles deformation and CFL formation. In a hyperbolic-shaped microchannel, the total Hencky strain experienced by a fluid element along the centreline of the channel can be determined by $\varepsilon_{H}=\ln \left(\mathrm{W}_{1} / \mathrm{W}_{2}\right)$, where $\mathrm{W}_{1}$ corresponds to the wide dimension of the converging channel whereas $W_{2}$ corresponds to the minimum contraction width (see Fig. 1) $[44,45]$. In the present study, all the flow experiments were performed with a $\varepsilon_{H}$ of about 3.5 .

Flows of cells and microparticles through microfluidic contractions generate complex flow phenomena, i. e., the fluid flow experiences a reduction in the cross-sectional area, which generates strongly-converging flows as the fluid pass through the contraction. The schematic illustration in Fig. 1 shows the fluid flow behaviour in a microfluidic hyperbolic-shaped contraction through which the RBCs and microparticles were tested. In general, the flow exhibits mixed kinematics with strong extensional flow along the centreline and shear flow close to the walls. For this latter region, the shear rate can be define as $\dot{\gamma}=\frac{d u_{x}}{d_{y}}$, where $u_{x}$ refers to the cell/particle axial velocity. The major advantage of a hyperbolic-shaped contraction is the ability to generate strong extensional flows with a homogeneous strain-rate along the centreline of the contraction. For this region, the strain rate can be define as $\dot{\varepsilon}=\frac{d u_{x}}{d_{x}}$. Due to these merits, during the last decade, several research works have measured the effects of the extensional flow in these kind of microchannels using blood analogue fluids and in vitro blood containing different kinds of blood cells [10,13,31,44-47].

\subsection{Microparticles production procedure}

The procedure, experimental setup and flow-focusing technique used in this study to generate the monodisperse PDMS microparticles were well explained and described in [7] and [48].

Once the monodisperse droplets are generated by the flowfocusing procedure, to get the solid PDMS particles from them, the emulsion of droplets was deposited in a glass optical cell [7]. This container was heated up at $70^{\circ} \mathrm{C}$ for $14 \mathrm{~h}$ with the heater of a common magnetic stirrer in order to conduct crosslinking and cure the PDMS droplets in the solution. Then, when the solution cooled down, it was diluted in water and the PDMS solid microparticles got separated from the glycerol and surfactant bulk by making the suspension pass through a porous glass microfiber filter of $1.2 \mu \mathrm{m}$ in pore size. After all the liquid has been suctioned by a pump, the filter was cleaned with a stream of 1-propanol to drag the microparticles to a petri dish. By letting the propanol evaporate, we obtain the dried PDMS microparticles in the petri dish and resuspend them in various media depending on the application. In the case of this work, they will be suspended in Dextran 40 .

To fabricate the flexible microparticles, we used a common transparent PDMS elastomer kit (Sylgard $® 186$ Silicone Elastomer Kit, Dow Corning) consisting of two parts: a PDMS precursor and a curing agent; and a black PDMS elastomer kit (Sylgard ${ }^{\circledR}$ 170, Silicone Elastomer Kit, Dow Corning) with a standardized ratio of mixture precursor/curing agent of 1:1. Three different samples were prepared with the transparent PDMS, by varying the mixture proportions as $10: 1,8: 2$ and $6: 4$. Also, based on the work performed by Anes et al. [48], the transparent PDMS mixed at a ratio of 10:1 was dyed with a red pigment for silicone (paste of PP SIL RO-1, Plastiform) at $20 \mathrm{wt} \%$, in order to produce coloured microparticles.

\subsection{Working fluids}

Several suspensions of PDMS microparticles in Dextran 40 were obtained, as summarized in Table 1. Rheological measurements and flow visualizations were performed on them to investigate whether the produced PDMS suspensions have flow properties similar to in vitro blood experiments.

Moreover, the same measurements were performed for additional working fluids (blood suspensions, see Table 1) in order to compare the behaviour between PDMS microparticles and healthy and pathological RBCs. Note that the cured PDMS microparticles are in all cases approximately the size of human RBCs $(\sim 8 \mu \mathrm{m})$. The pathological RBCs were obtained from a patient diagnosed with Diabetes Mellitus type II. 
b)

a)

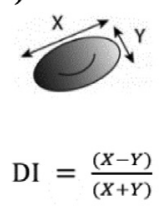

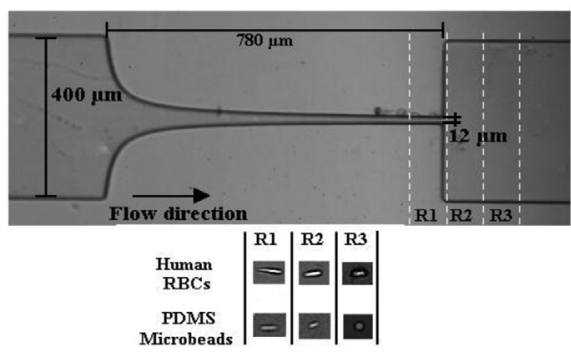

Fig. 2. a) Schematic representation of the deformation index (DI), and b) illustration of the three regions R1, R2 and R3 to evaluate the deformation index of the RBCs and PDMS microparticles, when flowing through the PDMS microchannel. Typical deformations for each region are shown at the bottom.

More detailed information regarding the preparation of the in vitro blood samples can be found at [49].

\subsection{Flow visualizations and microchannel geometry}

RBCs and microparticles suspensions were made to flow through a microchannel with a hyperbolic-shaped contraction. This shape produces a controlled quasi-homogeneous extensional flow field through the contraction (constant strain rate), thus allowing to elongate the flexible microparticles/cells. The microentities were visualized with a high-speed video microscopy system that combined an inverted microscope (IX71, Olympus, Japan) with a highspeed camera (Fastcam SA3, Photron, USA), as detailed in [13]. The flow was imposed by a syringe-pump (PHD Ultra, Harvard Apparatus, USA) at the flow rates of 5,8 and $10 \mu \mathrm{L} / \mathrm{min}$.

Fig. 2b shows the main dimensions of the used microchannel, where the depth had a constant value of about $15 \mu \mathrm{m}$. The three selected regions to obtain the deformability measurements are marked as R1, R2 and R3. By recording several highspeed videos of the flow and evaluating them with the image analysis strategy from [50] and the software Image J (NIH), we measured the deformation index of both microparticles and RBCs. The deformation index (DI) is defined in the equation (see Fig. 1a), where $X$ and $Y$ are respectively the major and minor axis of the cells or microparticles [10]. The DI values in region 1 (R1), i. e., the narrowest region of the hyperbolic contraction, are expected to be the highest. Additionally, measurements were performed in region 2 (R2) and region 3 (R3), where there is an abrupt expansion after the hyperbolic-shaped contraction and the microparticles and cells tend to recover their original shape.

The microchannel also allows to measure the microparticle- and cell-free layer when visualizing the flow, based on the work of Rodrigues et al. [51], they have reported that hyperbolic contractions have a strong effect on the thickness of the downstream CFL.

\subsection{Rheological characterization}

The rheological characterization of all the working fluids was performed in a stress-controlled rheometer (Bohlin CVO, Malvern) by using a cone-plate geometry with $55 \mathrm{~mm}$ in diameter and a gap size of $30 \mu \mathrm{m}$. The steady shear flow measurements were performed at $22{ }^{\circ} \mathrm{C}$ in a range of shear rates of $1 \mathrm{~s}^{-1} \leq \dot{\gamma} \leq 10000 \mathrm{~s}^{-1}$. For each working fluid, suspensions of either RBCs or PDMS microparticles, the measurements were performed three times with a fresh new sample of each, in order to corroborate the reproducibility.

\section{Results and discussion}

\subsection{Deformation index (DI)}

Suspensions of PDMS microparticles for different mixture ratios were visualized when flowing through the microchannel represented in Fig. 2b. Several videos were recorded at the flow rates of 5,8 and $10 \mu \mathrm{L} / \mathrm{min}$ with the aim to analyse the ability of the PDMS microparticles to deform and elongate when passing through the small microcontraction ( $12 \mu \mathrm{m})$, thus quantitatively evaluating their ability to mimic the deformation of human RBCs, either healthy or pathological.

Fig. 3 shows that all PDMS mixtures used to fabricate the microparticles present some degree of deformability at region R1. This demonstrates that PDMS is a good option to develop flexible microparticles. It is also clear that their deformation increases with the flow rate, as it happens with RBCs, presenting, both microparticles and RBCs, the highest DI values for the flow rate of $10 \mu \mathrm{L} / \mathrm{min}$.

As the microparticles flow out of the hyperbolic contraction, an abrupt expansion occurs, and R2 and R3 work as recuperation regions. DI at these regions are much lower for the PDMS microparticles than for RBCs. All PDMS samples present a similar and fast retrieval of their original spherical shape, resulting in a DI value close to zero; whereas RBCs, due to their unique biomechanical properties, show a higher recovery time when compared to the PDMS microparticles [31,33,52].

When comparing the behaviour of the different PDMS samples, there is not a significant variation between the transparent mixtures (ratios of 10:1, 8:2 and 6:4), since their DI values are all similar for each flow rate. On the contrary, the pigmented samples (red-dyed 10:1 PDMS and commercial black PDMS) happen to be much less deformable than the transparent ones. This effect was already been observed by the work performed by Khare et al. [53], where they have found a reduction in the Young's Modulus of PDMS micropillars due to the addition of a dye.

Clearly, DI measurements for the pigmented samples are far from the human RBCs values, either the healthy or the pathological ones. Hence, even if these coloured particles enhance contrast and visualization [48], they do not seem to be adequate to represent RBCs in the development of particulate analogue fluids.

On the other hand, the transparent PDMS particles present, in R1, a midway DI value between healthy and pathological RBCs, thus being able to mimic RBCs with a certain degree of disease, for example an intermediate status of Diabetes Mellitus type II.

To the best of the authors' knowledge, these proposed particles show the closest behaviour to human RBCs in terms of deformability. Therefore, they seem to be the best candidates for the moment to qualitatively mimic them and be integrated in blood analogue fluids.

\subsection{Steady shear rheology}

As previously reported by Muñoz-Sánchez et al. [7], a suspension of 10:1 PDMS microparticles with an average diameter of $5.87 \mu \mathrm{m}$ demonstrated to have a close behaviour to a suspension of ovine RBCs (mean diameter of $5 \mu \mathrm{m}$ ), when comparing their steady shear viscosity curves. In this study, we obtained, under steadystate experiments, the flow behaviour of the suspensions with the most deformable microparticles: those made of transparent PDMS (ratios 10:1, 8:2 and 6:4), to be compared against human RBCs.

Since the disadvantage of the production method is the low throughput, with the aim to increase the concentration of particles in the suspension, we prepared a final working fluid with all the microparticles that had a similar deformability, i.e. we merged transparent PDMS microparticles of 10:1, 8:2 and 6:4 ratios and 
a)

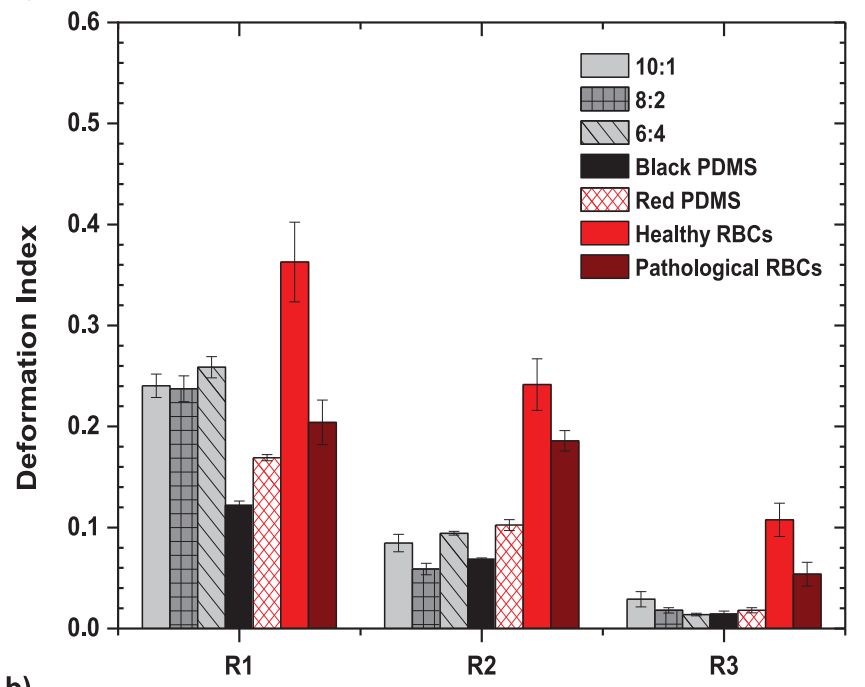

b)

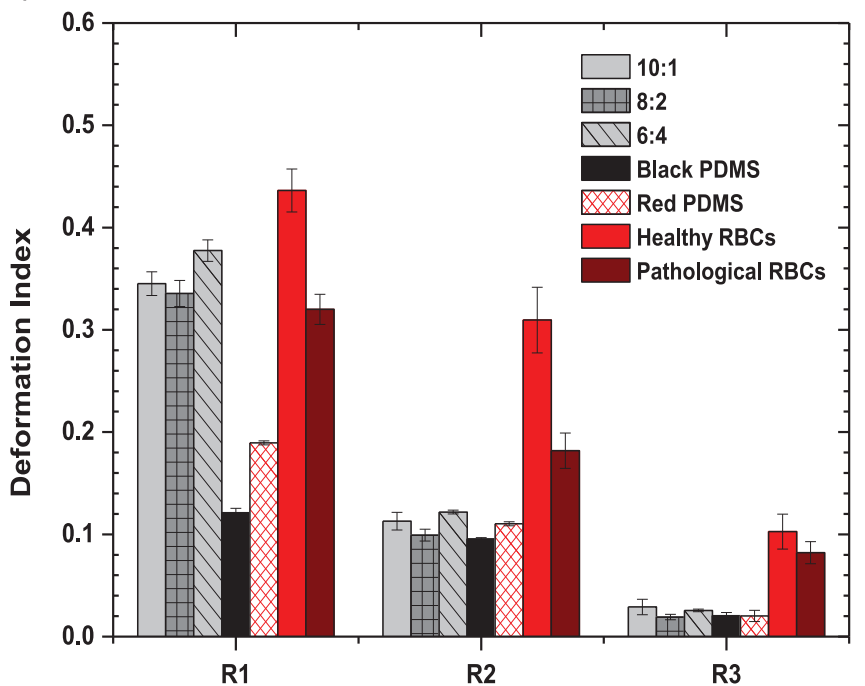

c)

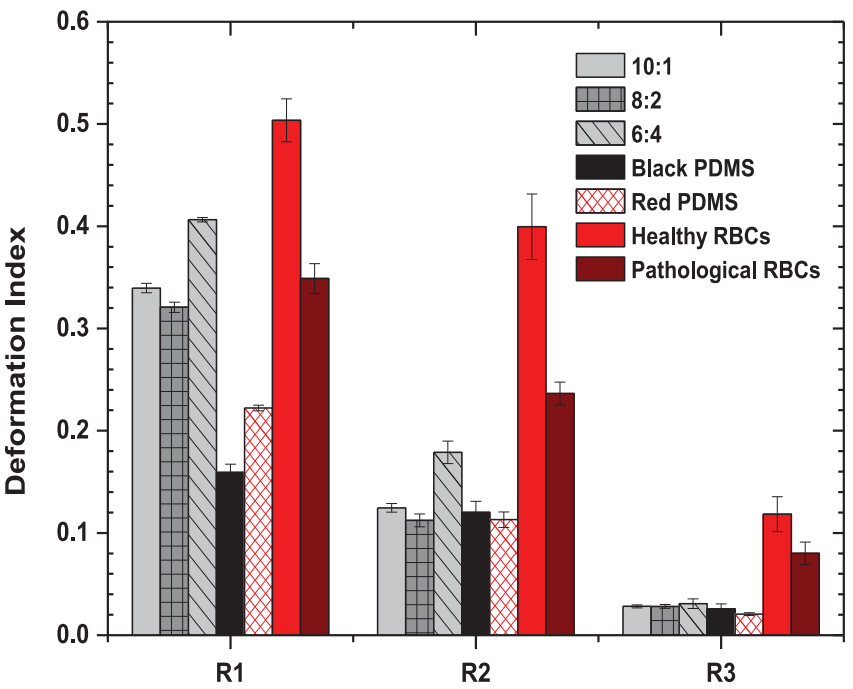

Fig. 3. Deformability results at the regions in Fig. $1 \mathrm{~b}$ for all the produced PDMS microparticles and RBCs at the flow rates of: a) $5 \mu \mathrm{L} / \mathrm{min}$; b) $8 \mu \mathrm{L} / \mathrm{min}$ and c) $10 \mu \mathrm{L} / \mathrm{min}$. The error bars represent the confidence interval at a level of $95 \%$ for $n=100$ (100 microparticles were measured for each sample).

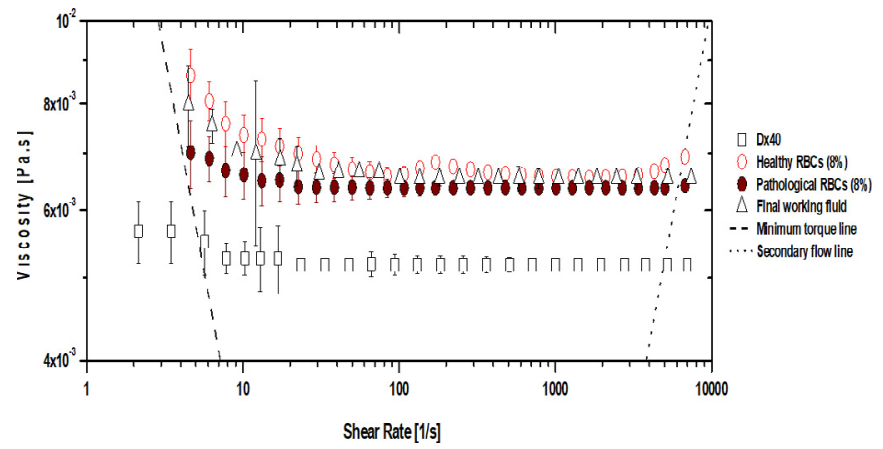

Fig. 4. Steady shear viscosity curves for the suspending medium (Dx 40) and suspensions of different particles at a concentration of $8 \% \mathrm{vol}$ : healthy RBCs, pathological RBCs and final working fluid (PDMS microparticles of ratios 10:1, 8:2 and 6:4). The error bars represent the confidence interval at a level of $95 \%$.

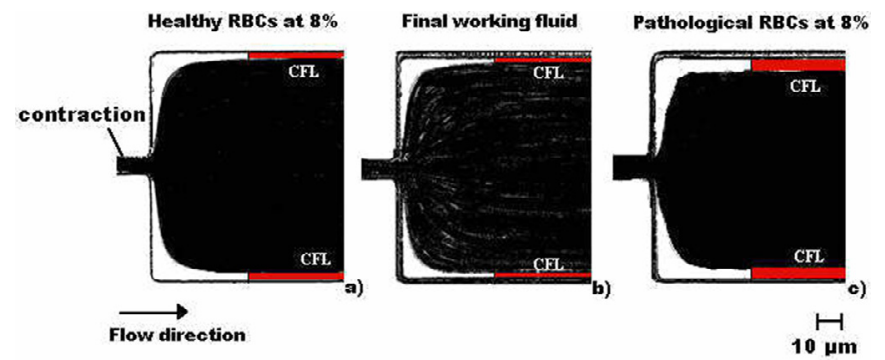

Fig. 5. CFL marked in red in the microchannel at the flow rate of $8 \mu \mathrm{L} / \mathrm{min}$ for: a) suspension of healthy RBCs; b) final working fluid; c) suspension of pathological RBCs. Detailed information about image processing in ImageJ can be found in [31].

suspended them all in Dx 40. The same carrier fluid was used to create the suspensions of human RBCs, both the healthy and the pathological, at close concentration of the PDMS microparticles $(8 \% \mathrm{vol})$.

Two different tendencies can be clearly distinguished in the viscosity curves of Fig. 4. On the one hand, the sample containing only the suspending medium (Dx 40) presents a constant viscosity over all the shear rates. On the contrary, the viscosity curves of the particulate suspensions show a smooth shear-thinning behaviour at small shear rates and constant viscosity (Newtonian behaviour) at high shear rates. When comparing both tendencies, we notice that the increase in suspension concentration (due to the addition of either microparticles or RBCs) promotes an increment of the viscosity in the Newtonian plateau over that of the suspending medium.

A closer look into the low shear-rate range reveals that the suspension of pathological RBCs has a smaller shear-thinning degree than the healthy RBCs. The final working fluid shows an intermediate behaviour between both the healthy and the pathological RBCs.

It is clear from the results that the sample containing PDMS microparticles shows a good agreement with the rheological properties of the in vitro RBCs samples, with an intermediate shearthinning degree that makes these microparticles an ideal candidate to mimic suspensions of RBCs with a certain degree of pathology.

\subsection{Cell-free layer visualization}

We also observed whether a cell-free layer, or -better called for the final working fluid- a 'microparticle-free layer', was formed close to the walls of the microchannel. The microchannel used is the same to measure the deformation indexes, shown in Fig. $2 \mathrm{~b}$.

In the case of the present work, Fig. 5 shows that the suspension of PDMS microparticles develops, downstream of a contraction and close to the microchannel walls, a clear region with- 


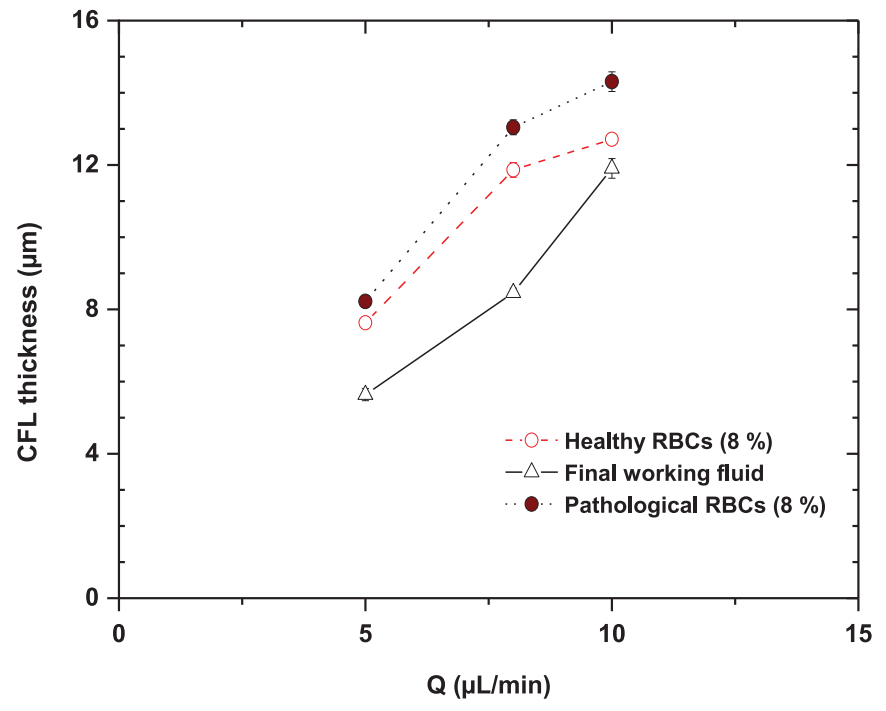

Fig. 6. CFL thickness for the different suspensions at all the flow rates: 5,8 and $10 \mu \mathrm{L} / \mathrm{min}$. The error bars represent the confidence interval at a level of $95 \%$.

out microparticles, similarly to what occurs for human RBCs (both healthy and pathological).

In addition, this microparticle-free layer grows with the increase of the flow rate, in the same trend as the suspensions of RBCs (see Fig. 6).

\section{Conclusions}

In this work, we have studied PDMS microparticles with diameters below $10 \mu \mathrm{m}$ fabricated by a flow-focusing technique with different crosslinking ratios and pigmentation. The flow behaviour of suspensions created with such microparticles has been compared against suspensions of human RBCs, both healthy and pathological, all of them reproducing several microcirculation phenomena.

For the first time, the Deformation Index (DI) has been measured and compared for different PDMS microparticles. By visualizing the flow through a hyperbolic-shaped contraction, we observed that all PDMS microparticles present some ability to elongate when passing through the smallest dimension of the microchannel. The samples with the highest DI were the transparent PDMS mixtures. All the crosslinking ratios showed comparable deformability among them, with a similar value to human RBCs. In particular, transparent PDMS microparticles yielded a midway DI value between healthy and pathological RBCs. Nevertheless, pigmented microparticles show reduced DI values, so they are not valid to replicate RBCs. Since colour enhances flow visualization, it would be desirable to further develop this type of particles in terms of deformability.

By means of rheological measurements, we also verified that the final working fluid (formed of transparent PDMS microparticles) is able to reproduce the viscosity curve of human RBCs suspensions, showing an intermediate shear-thinning degree between healthy and pathological RBCs. Hence, regarding deformability values and viscosity curves, transparent PDMS microparticles emerge as good candidates to mimic diseased RBCs, such as those with an intermediate status of Diabetes Mellitus type II.

In addition, the final working fluid mimics another phenomenon found for in vivo and in vitro microcirculation of RBCs: the cell-free layer (CFL). The flow visualization shows that this fluid forms a layer free of microparticles downstream of a contraction, in the same way that suspensions of human RBCs (either healthy or pathological) do, leaving a cell-depleted plasma region near the walls of the microchannel.
As future directions, we propose a scale-up of the production in order to obtain a higher concentration of microparticles, thus allowing more detailed studies and applications, and a further development of the coloured microparticles, since colouring is a way to improve flow visualization of particles within microchannels.

To the best of the authors' knowledge, the developed PDMS microparticles seem to be the best candidates for the moment to qualitatively mimic the deformability of human RBCs. Our results support their great potential to develop particulate blood analogue fluids with hemorheological behaviour similar to in vitro blood suspensions.

\section{Declaration of Competing Interest}

The authors declare no conflict of interest.

\section{Acknowledgements}

The authors acknowledge the financial support provided by Fundação para a Ciência e a Tecnologia (FCT), through the projects UID/EMS/04077/2019, UID/EMS/00532/2019, POCI-01-0145-FEDER016861 (with associated reference PTDC/QEQ-FTT/4287/2014), NORTE-01-0145-FEDER-029394 and NORTE-01-0145-FEDER-030171, funded by COMPETE2020, NORTE 2020, PORTUGAL 2020 and FEDER. Financial support from the Spanish Ministry of Science and Education (grant no. DPI2016-78887), Junta de Extremadura (grants no. GR15014 and IB18005, partially financed by FEDER funds), and "la Caixa" Foundation (ID 100010434, grant LCF/BQ/ES14/10320005) is gratefully acknowledged too. Rui Lima also acknowledges the fellowship SFRH/BSAB/135419/2017 granted by FCT.

\section{Supplementary materials}

Supplementary material associated with this article can be found, in the online version, at doi:10.1016/j.mechrescom.2019. 103399.

\section{References}

[1] I.D. Johnston, D.K. McCluskey, C.K.L. Tan, M.C. Tracey, Mechanical characterization of bulk Sylgard 184 for microfluidics and microengineering, J. Micromech Microeng. 24 (3) (2014) 35017, doi:10.1088/0960-1317/24/3/035017.

[2] H. Tsutsui, C. Ho, Cell separation by non-inertial force fields in microfluidic systems, Mech. Res. Commun. 36 (2009) 92-103, doi:10.1016/j.mechrescom. 2008.08.006.

[3] Z. Wang, A.A. Volinsky, N.D. Gallant, Crosslinking effect on polydimethylsiloxane elastic modulus measured by custom-built compression instrument, J Appl. Polym. Sci. 131 (22) (2014) 41050, doi:10.1002/app.41050.

[4] V. Faustino, S.O. Catarino, R. Lima, G. Minas, Biomedical microfluidic devices by using low-cost fabrication techniques: a review, J. Biomech. 49 (11) (2016) 2280-2292, doi:10.1016/j.jbiomech.2015.11.031.

[5] C. Cardoso, C.S. Fernandes, R. Lima, J. Ribeiro, Biomechanical analysis of PDMS channels using different hyperelastic numerical constitutive models, Mech. Res. Commun. 90 (2018) 26-33, doi:10.1016/j.mechrescom.2018.04.007.

[6] Y.H. Choi, K.H. Chung, H.B. Hong, W.S. Lee, Production of PDMS microparticles by emulsification of two phases and their potential biological application, Int. J. Polym. Mater. Polym. Biomater. 67 (11) (2017) 686-692, doi:10.1080/ 00914037.2017.1375494.

[7] B.N. Muñoz-Sánchez, S.F. Silva, D. Pinho, E.J. Vega, R. Lima, Generation of micro-sized PDMS particles by a flow focusing technique for biomicrofluidics applications, Biomicrofluidics 10 (1) (2016) 14122, doi:10.1063/1.4943007.

[8] A. Ghanbari, V. Nock, S. Johari, R. Blaikie, X. Chen, W. Wang, A micropillarbased on-chip system for continuous force measurement of C. elegans, J. Micromech. Microeng. 22 (9) (2012) 095009, doi:10.1088/0960-1317/22/9/ 095009.

[9] T. Fujiwara, I.D. Johnston, M.C. Tracey, C.K.L. Tan, Increasing pumping efficiency in a micro throttle pump by enhancing displacement amplification in an elastomeric substrate, J. Micromech. Microeng. 20 (6) (2010) 065018 doi:10.1088/0960-1317/20/6/065018.

[10] D. Bento, R. Rodrigues, V. Faustino, D. Pinho, C. Fernandes, A. Pereira, V. Garcia, J. Miranda, R. Lima, Deformation of red blood cells, air bubbles, and droplets in microfluidic devices: flow visualizations and measurements, Micromachines 9 (4) (2018) 151, doi:10.3390/mi9040151. 
[11] A. Rubio, V. Faustino, M.G. Cabezas, R. Lima, E.J. Veja, Fire-shaped cylindrical glass micronozzles to measure cell deformability, J. Micromech. Microeng. 29 (2019) 105001 https://doi.org/10.1088/1361-6439/ab3183.

[12] S. Tripathi, Y.V.B. Varun Kumar, A. Prabhakar, S.S. Joshi, A. Agrawal, Passive blood plasma separation at the microscale: a review of design principles and microdevices, J. Micromech. Microeng. 25 (2015) 083001, doi:10.1088/ 0960-1317/25/8/083001.

[13] R.O. Rodrigues, D. Pinho, V. Faustino, R. Lima, A simple microfluidic device for the deformability assessment of blood cells in a continuous flow, Biomed. Microdevices 17 (6) (2015) 108, doi:10.1007/s10544-015-0014-2.

[14] J. Zhu, J. Shang, Y. Jia, K. Liu, D. Brenner, Q. Lin, Physical modulation based cell manipulation in microfluidic devices, in: 8th Annu. IEEE Int. Conf. Nano/Micro Eng. Mol. Syst., Suzhou, China, IEEE, 2013, pp. 1226-1229, doi:10.1109/NEMS. 2013.6559940.

[15] C.N. Baroud, F. Gallaire, R. Dangla, Dynamics of microfluidic droplets, Lab. Chip 10 (16) (2010) 2032-2045, doi:10.1039/C001191F.

[16] T.P. Lagus, J.F. Edd, A review of the theory, methods and recent applications of high-throughput single-cell droplet microfluidics, J. Phys. D. Appl. Phys. 46 (11) (2013) 114005, doi:10.1088/0022-3727/ 46/11/114005.

[17] J. Xu, D. Attinger, Drop on demand in a microfluidic chip, J. Micromech. Microeng. 18 (6) (2008) 065020, doi:10.1088/0960-1317/18/6/065020.

[18] S.L. Anna, N. Bontoux, H.A. Stone, Formation of dispersions using "flow focusing" in microchannels, Appl. Phys. Lett. 82 (3) (2003) 364-366, doi:10.1063/1. 1537519.

[19] J. Carneiro, E. Doutel, J.B.L.M. Campos, J.M. Miranda, PDMS droplet formation and characterization by hydrodynamic flow focusing technique in a PDMS square microchannel, J. Micromech. Microeng. 26 (10) (2016) 105013, doi:10. 1088/0960-1317/26/10/105013.

[20] A.S. Utada, A. Fernandez-Nieves, H.A. Stone, D.A. Weitz, Dripping to jetting transitions in coflowing liquid streams, Phys. Rev. Lett. 99 (9) (2007) 94502, doi:10.1103/PhysRevLett.99.094502.

[21] D. Bento, L. Sousa, T. Yaginuma, V. Garcia, R. Lima, J.M. Miranda, Microbubble moving in blood flow in microchannels: effect on the cell-free layer and cell local concentration, Biomed. Microdevices 19 (1) (2017) 6, doi:10.1007/ s10544-016-0138-z.

[22] J. Carneiro, E. Doutel, J.B.L.M. Campos, J.M. Miranda, Visualization of PDMS microparticles formation for biomimetic fluids, Micro Nanosyst. 7 (3) (2015) 154158, doi:10.2174/1876402908666160106000558.

[23] T. Nisisako, T. Torii, T. Higuchi, Droplet formation in a microchannel network, Lab. Chip 2 (0) (2002) 24-26, doi:10.1039/B108740C.

[24] K. Jiang, P.C. Thomas, S.P. Forry, D.L. DeVoe, S.R. Raghavan, Microfluidic synthesis of monodisperse PDMS microbeads as discrete oxygen sensors, Soft Matter. 8 (4) (2012) 923-926, doi:10.1039/C2SM06685H.

[25] D. Bento, C.S. Fernandes, J.M. Miranda, R. Lima, In vitro blood flow visualizations and cell-free layer (CFL) measurements in a microchannel network, Exp. Therm. Fluid Sci. 109 (2019) 109847 https://doi.org/10.1016/j.expthermflusci. 2019.109847.

[26] H. Sun, M. Björnmalm, J. Cui, E.H.H. Wong, Y. Dai, Q. Dai, G.G. Qiao, F. Caruso, Structure governs the deformability of polymer particles in a microfluidic blood capillary model, ACS Macro Lett. 4 (11) (2015) 1205-1209, doi:10.1021/ acsmacrolett.5b00591.

[27] T.W. Secomb, A.R. Pries, Blood viscosity in microvessels: experiment and theory, Comptes Rendus Phys. 14 (6) (2013) 470-478, doi:10.1016/j.crhy.2013.04. 002.

[28] R. Lima, T. Ishikawa, Y. Imai, T. Yamaguchi, Blood flow behavior in microchannels: past, current and future trends, in: R. Lima, T. Ishikawa, Y. Imai, T. Yamaguchi (Eds.), Single Two-Phase Flows Chem. Biomed. Eng., Bentham Science Publishers, 2012, pp. 513-547, doi:10.2174/978160805295011201010513.

[29] N. Maeda, Erythrocyte rheology in microcirculation, Jpn. J. Physiol. 46 (1) (1996) 1-14, doi:10.2170/jjphysiol.46.1.

[30] J. Calejo, D. Pinho, F. Galindo-Rosales, R. Lima, L. Campo-Deaño, Particulate blood analogues reproducing the erythrocytes cell-free layer in a microfluidic device containing a hyperbolic contraction, Micromachines 7 (1) (2016) 4, doi: $10.3390 / \mathrm{mi} 7010004$.

[31] D. Pinho, L. Campo-Deaño, R. Lima, F.T. Pinho, In vitro particulate analogue fluids for experimental studies of rheological and hemorheological behavior of glucose-rich RBC suspensions, Biomicrofluidics 11 (5) (2017) 54105, doi:10. 1063/1.4998190.

[32] N. Doshi, A.S. Zahr, S. Bhaskar, J. Lahann, S. Mitragotri, Red blood cellmimicking synthetic biomaterial particles, Proc. Natl. Acad. Sci. USA 106 (51) (2009) 21495-21499, doi:10.1073/pnas.0907127106.
[33] S. She, Q. Li, B. Shan, W. Tong, C. Gao, Fabrication of red-blood-cell-like polyelectrolyte microcapsules and their deformation and recovery behavior through a microcapillary, Adv. Mater. 25 (40) (2013) 5814-5818, doi:10.1002/ adma.201302875

[34] X. Ju, X. Wang, Z. Liu, R. Xie, W. Wang, L. Chu, Red-blood-cell-shaped chitosan microparticles prepared by electrospraying, Particuology 30 (2017) 151-157, doi:10.1016/j.partic.2016.05.011.

[35] K. Chen, T.J. Merkel, A. Pandya, M.E. Napier, J.C. Luft, W. Daniel, S. Sheiko, J.M. DeSimone, Low modulus biomimetic microgel particles with high loading of hemoglobin, Biomacromolecules 13 (9) (2012) 2748-2759, doi:10.1021/ bm3007242.

[36] J. Cui, M. Björnmalm, K. Liang, C. Xu, J.P. Best, X. Zhang, F. Caruso, SuperSoft hydrogel particles with tunable elasticity in a microfluidic blood capillary model, Adv. Mater. 26 (43) (2014) 7295-7299, doi:10.1002/adma.201402753.

[37] A.C. Anselmo, M. Zhang, S. Kumar, D.R. Vogus, S. Menegatti, M.E. Helgeson, S. Mitragotri, Elasticity of nanoparticles influences their blood circulation, phagocytosis, endocytosis, and targeting, ACS Nano 9 (3) (2015) 3169-3177, doi:10.1021/acsnano.5b00147.

[38] L. Zhang, Z. Cao, Y. Li, J.-.R. Ella-Menye, T. Bai, S. Jiang, Softer Zwitterionic Nanogels for longer circulation and lower splenic accumulation, ACS Nano 6 (8) (2012) 6681-6686, doi:10.1021/nn301159a.

[39] T.J. Merkel, S.W. Jones, K.P. Herlihy, F.R. Kersey, A.R. Shields, M.E. Napier, J.C. Luft, H. Wu, W.C. Zamboni, A.Z. Wang, J.E. Bear, J.M. DeSimone, Using mechanobiological mimicry of red blood cells to extend circulation times of hydrogel microparticles, Proc. Natl. Acad. Sci. USA 108 (2) (2011) 586-591, doi:10.1073/pnas.1010013108.

[40] N. Vilanova, C. Rodríguez-Abreu, A. Fernández-Nieves, C. Solans, Fabrication of novel silicone capsules with tunable mechanical properties by microfluidic techniques, ACS Appl. Mater. Interfaces 5 (11) (2013) 5247-5252, doi:10.1021/ am4010896.

[41] H.A Stone, Introduction to fluid dynamics for microfluidic flows, in: H. Lee, R.M. Westervelt, D. Ham (Eds.), CMOS Biotechnology. Series on Integrated Circuits and Systems, Springer, Boston, 2007.

[42] M. Abkarian, M. Faivre, R. Horton, K. Smistrup, C.A. Best-Popescu, H.A. Stone Cellular-scale hydrodynamics, Biomed. Mater. 3 (3) (2008) 034011.

[43] H. Bruus, Theoretical Microfluidics, Theoretical Microfluidics, Oxford University Press, Oxford, 2008.

[44] S.S. Lee, Y. Yim, K.H. Ahn, S.J. Lee, Extensional flow-based assessment of red blood cell deformability using hyperbolic converging microchannel, Biomed. Microdevices 11 (5) (2009) 1021-1027.

[45] P.C. Sousa, F. T.Pinho, M.S.N. Oliveira, M. Alves, Extensional flow of blood analog solutions in microfluidic devices, Biomicrofluidics 5 (1) (2011) 014108.

[46] D.A.M. Carvalho, A.R.O. Rodrigues, V. Faustino, D. Pinho, E.M.S. Castanheira, R. Lima, Microfluidic deformability study of an innovative blood analogue fluid based on giant unilamellar vesicles, J. Funct. Biomater. 9 (4) (2018) 70.

[47] R.O. Rodrigues, M. Bañobre-López, J. Gallo, P.B. Tavares, A.M.T. Silva, R. Lima, H.T. Gomes, Haemocompatibility of iron oxide nanoparticles synthesized for theranostic applications: A high-sensitivity microfluidic tool, J. Nanopart. Res. 18 (194) (2016), doi:10.1007/s11051-016-3498-7.

[48] C.F. Anes, D. Pinho, B.N. Muñoz-Sánchez, E.J. Vega, R. Lima, Shrinkage and colour in the production of micro-sized PDMS particles for microfluidic applications, J. Micromech. Microeng. 28 (7) (2018) 075002, doi:10.1088/1361-6439/ aab7b9.

[49] D. Pinho, R.O. Rodrigues, V. Faustino, T. Yaginuma, J. Exposto, R. Lima, Red blood cells radial dispersion in blood flowing through microchannels: the role of temperature, J. Biomech. 49 (11) (2016) 2293-2298, doi:10.1016/j.jbiomech. 2015.11.037.

[50] T. Yaginuma, M.S.N. Oliveira, R. Lima, T. Ishikawa, T. Yamaguchi, Human red blood cell behavior under homogeneous extensional flow in a hyperbolicshaped microchannel, Biomicrofluidics 7 (5) (2013) 054110, doi:10.1063/1. 4820414.

[51] R.O. Rodrigues, R. Lopes, D. Pinho, A.I. Pereira, V. Garcia, S. Gassmann, P.C. Sousa, R. Lima, In vitro blood flow and cell-free layer in hyperbolic microchannels: visualizations and measurements, BioChip J. 10 (1) (2016) 9-15, doi:10.1007/s13206-016-0102-2.

[52] G. Tomaiuolo, Biomechanical properties of red blood cells in health and disease towards microfluidics, Biomicrofluidics 8 (5) (2014) 051501, doi:10.1063/ 1.4895755

[53] S.M. Khare, A. Awasthi, V. Venkataraman, S.P. Koushika, Colored polydimethylsiloxane micropillar arrays for high throughput measurements of forces applied by genetic model organisms, Biomicrofluidics 9 (1) (2015) 014111, doi:10. $1063 / 1.4906905$. 